\title{
Perturbation analysis of an electrostatic Micro-Electro-Mechanical System (MEMS) subjected to external and non-linear parametric excitations
}

\author{
Abd-Elrehim M. Elnaggar, Atef F. El-Bassiouny, Gamal A. Mosa * \\ Department of Mathematics,Faculty of Science, Benha University, Egypt, B. O. 13518 \\ *Corresponding author E-mail: gamal.mosa10@gmail.com
}

\begin{abstract}
Copyright (C)2014 Elnaggar et. al. This is an open access article distributed under the Creative Commons Attribution License Creative Commons Attribution License, which permits unrestricted use, distribution, and reproduction in any medium, provided the original work is properly cited.
\end{abstract}

\begin{abstract}
Electrostatic micro-electro-mechanical system (MEMS) is a special branch with a wide range of applications in sensing and actuating devices in MEMS. In this paper the perturbation analysis of the electrostatically actuated MEMS resonant sensors which represented by a modified Duffing - Van der Pol equation subjected to weakly non-linear parametric and external excitations is studied by using a perturbation technique (multiple time scales). Harmonic resonance and subharmonic resonances of order $\left(\frac{1}{2}\right.$ and $\left.\frac{1}{3}\right)$ are investigated. For each resonances we obtain the modulation equations in the amplitude and phase, steady state solutions, frequency-response equations and stability conditions are determined. Effects of different parameters on the system behavior are investigated numerically. Results are presented graphically and discussion is provided.
\end{abstract}

Keywords: MEMS, Weakly non-linear dynamical systems, Micro-cantilever, Parametric and Forcing excitations.

\section{Introduction}

Electrostatic-driven microelectromechanical systems devices, in most cases, consist of couplings of such energy domains as electromechanics, optical electricity, thermoelectricity, and electromagnetism [1]. Electrostatic MEMS is an important branch with a wide range of applications including sensing devices and actuating devices in MEMS [2]. For example, the sensing devices such as capacitive accelerometers [3] and capacitive sensors [4]. In MEMS, electrostatic forces are often used to actuate microstructures, including switches [5,6], electrostatic motors [7,8], etc. The use of electrostatic actuation in MEMS is attractive because of the quite efficient, high energy densities and large forces available for MEMS devices in micro-scale [9].

A serious limitation on the use of these devices lies in the pull-in phenomenon [10-12], which is a structural instability resulting from the interaction between elastic and electrostatic forces. This instability results from the unbalance between the electric actuation and the mechanical restoring force leading the suspended microbeam to hit the stationary electrode underneath it causing stiction and short circuit problems and hence the failure in the devices function [13]. Keeping MEMS devices operating in a stable regime away from the pull-in instability limit has a crucial interest from design and commercialization point of view. Various works [14, 15] investigated the 
static pull-in phenomenon and performed techniques to predict its occurrence by determining the best conditions for which the system operate in a stable behavior. There are various methods can be used to drive MEM structures at resonance. The most commonly used method is the primary-resonance excitation, in which the frequency of the excitation is tuned closed to the fundamental natural frequency of the microstructure. Examples of this excitation in MEMS can be found in various applications, such as resonant sensors [16] and RF filters [17]. Jin and Wang [18] showed that driving a resonant microsensor with a subharmonic excitation of order one-half increased the signal-to-crosstalk ratio as compared to driving it at primary resonance. Younis et al. [16,19] used the method of multiple scales to study the response of an electrostatically actuated resonator to a primary resonance excitation. Also Abdel-Rahman and Nayfeh [20] studied a superharmonic resonance excitation of order two, and a subharmonic resonance excitation of order one-half. Furthermore, Nayfeh and Younis [21] investigated the dynamics of a MEMS resonator to a subharmonic resonance of order one-half and to a superharmonic resonance of order two. Zhang and Meng [22] analyzed the nonlinear dynamics of the electrostatically actuated resonant MEMS sensors under parametric excitation. Harmonic, sub-harmonic and super-harmonic resonance of weakly non-linear dynamical system subjected to external excitation, parametric excitation or both are investigated by Elnaggar et al. [24-27]. Batra et al. [28] and Kacem et al. [29-31] studied respectively nonlinear multi-physics models including both mechanical and electrostatic nonlinearities and the fringing field effect. A more comprehensive review up to 2010 about the work on the non-linear dynamics of micro resonators is presented in literature [32].

Zhang et al. $[22,23]$ approximate the electrostatic force and the squeeze film damping force in the equation of motion by working with first and second order expansion and $\mathrm{O}\left(\varepsilon^{3}\right)$ term is neglected based on the small displacement (amplitude) assumption. Also, use a combination of DC and $\mathrm{AC}$ voltages. The focus of this paper is actuation of high displacement (amplitude) vibrations in MEMS using a square rooted sinusoidal AC voltage signal only, So, the higher order expansion terms up to $O\left(\varepsilon^{6}\right)$ for the electrostatic force and up to $O\left(\varepsilon^{3}\right)$ for the squeeze film damping force are taken. This leads to the mathematical model of the equation of motion is of the type generalized modified Duffing - Van der Pol equation subjected to weakly non-linear parametric and external excitations. In this paper harmonic resonance and subharmonic resonances of order $\left(\frac{1}{2}\right.$ and $\left.\frac{1}{3}\right)$ are investigated by applying the method of multiple scales. For each type of resonance we determine an approximate solution, steady-state solution, frequency response equation and stability of the steady-state solution. Frequency response curves are plotted in which solid curve represent stable solutions and dashed curve represent unstable solutions. Finally discussion for the figures is given.

\section{Formulation of the problem and perturbation analysis}

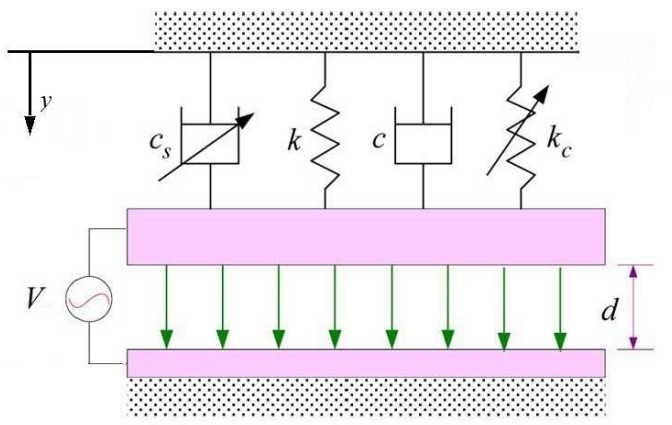

Fig.1: The diagram of the dynamical model of the problem

The governing equation of motion for a simplified dynamic system of the micro-cantilever beam in MEMS shown in (fig. 1) is represented by the following nonlinear second order differential equation [22]

$m \ddot{y}+c \dot{y}+k y+k_{c} y^{3}=F_{e}(y, t)+F_{s}(y, \dot{y})$

where $\left({ }^{\cdot}\right)$ denotes the derivative with respect to $t, y$ is the vertical displacement of the micro-cantilever relative to the origin of the fixed plate, $m$ is the mass, $k$ and $k_{c}$ are the coefficients of linear and non-linear spring stiffness , $c$ is the coefficient of linear damping and $F_{s}$ and $F_{e}$ are the squeeze film damping force and nonlinear electrostatic force, respectively. 
The electrostatic force, $F_{e}$, between the capacitor plates (the fixed plate and the movable plate) generated by applying a voltage $V(t)$, can be expressed as

$F_{e}=\frac{\epsilon_{0} A}{2} \frac{V^{2}(t)}{(d-y)^{2}}$

where $\epsilon_{0}$ is the absolute dielectric constant of vacuum, $A$ the overlapping area between the two plates, $d$ is the gap between them, and the force due to electrostatic interactions has a square dependence on the voltage applied, in order to isolate the parametric effects from harmonic effects, we can use a square rooted sinusoidal voltage signal $V=\left[V_{0} \cos (\omega t)\right]^{1 / 2}[33]$. Expanding the nonlinear electrostatic force $F_{e}$ by Taylor series with respect to the equilibrium position, i.e., $y_{0}=0$, gives

$\frac{\epsilon_{0} A}{2} \frac{V^{2}(t)}{(d-y)^{2}}=\frac{\epsilon_{0} A}{2} \frac{V^{2}(t)}{d^{2}}\left(1+\frac{2}{d} y+\frac{3}{d^{2}} y^{2}+\frac{4}{d^{3}} y^{3}+\frac{5}{d^{4}} y^{4}+\frac{6}{d^{5}} y^{5}+\frac{7}{d^{6}} y^{6}+\cdots\right)$.

Viscous damping of micro structures vibrating in air in the narrow gap between two electrodes is dominated by squeeze film damping. Starr [34] presented the expression of the damping force for a rectangular plate of dimension as

$F_{s}(y, \dot{y})=-\frac{c_{s}}{(d-y)^{3}} \dot{y}$

Expanding the squeeze film damping force $F_{s}$ by Taylor series, yields

$-\frac{c_{s}}{(d-y)^{3}} \dot{y}=-\frac{c_{s}}{d}\left(1+\frac{3}{d} y+\frac{6}{d^{2}} y^{2}+\frac{10}{d^{3}} y^{3}+\cdots\right) \dot{y}$

Substituting equations (3) and (5) into equation (1),

$\ddot{y}+\omega_{0}^{2} y+\varepsilon \lambda y^{3}+\varepsilon\left[\mu_{0}+\mu_{1}\left(1+3 y+6 y^{2}+10 y^{3}\right)\right] \dot{y}-\varepsilon F\left(2 y+3 y^{2}+4 y^{3}+5 y^{4}+6 y^{5}+7 y^{6}\right) \cos (\Omega t)=\varepsilon F \cos (\Omega t)$

where $\quad \omega_{0}=\sqrt{\frac{k}{m}}, \quad \varepsilon \lambda=\frac{k_{c} d^{2}}{m}, \quad \epsilon \mu_{0}=\frac{c}{m}, \quad \varepsilon \mu_{1}=\frac{c_{s}}{m d^{2}} \quad$ and $\quad F=\frac{\varepsilon_{0} A V_{0}^{2}}{2 m d^{3}}$

Equation (6) represents the modified Duffing - Van der Pol equation subjected to weakly non-linear parametric and forcing excitations. Here, $F$ and $\Omega$ are the amplitude and the frequency of parametric and external excitations, $\mu_{0}$ and $\mu_{1}$ are the coefficients of viscous linear and nonlinear damping terms, $\omega_{0}$ is the natural frequency and $\lambda$ is the cubic nonlinear stiffness. form

Using the method of Multiple Scales (Nayfeh [35]), we get a first order uniform solution of equation (6) in the

$y(t ; \varepsilon)=y_{0}\left(T_{0}, T_{1}\right)+\varepsilon y_{1}\left(T_{0}, T_{1}\right)+\ldots$,

where $T_{0}=t$ is the first scale associated with changes occurring at the frequencies $\omega_{0}$ and $\Omega$, and $T_{1}=\varepsilon t$ is a slow scale associated with modulations in the amplitude. In terms of $T_{1}$, the time derivatives become

$\frac{d}{d t}=D_{0}+\varepsilon D_{1}+\ldots \quad \quad \quad \frac{d^{2}}{d t^{2}}=D_{0}^{2}+2 \varepsilon D_{0} D_{1}+\ldots$

where $D_{n}=\frac{\partial}{\partial T_{n}}$. Substituting equations (7) and (8) into equation (6) and equating coefficients of like powers of $\varepsilon$ one obtains

$D_{0}^{2} y_{0}+\omega_{0}^{2} y_{0}=0$

$\begin{aligned} D_{0}^{2} y_{1}+\omega_{0}^{2} y_{1}= & -\lambda y_{0}^{3}-\left(\mu_{0}+\mu_{1}+3 \mu_{1} y_{0}+6 \mu_{1} y_{0}^{2}+10 \mu_{1} y_{0}^{3}\right) D_{0} y_{0}+2 D_{0} D_{1} y_{0} \\ & \left.+F\left(1+2 y_{0}+3 y_{0}^{2}+4 y_{0}^{3}+5 y_{0}^{4}+6 y_{0}^{5}+7 y_{0}^{6}\right) \cos \left(T_{0} \Omega\right)\right)\end{aligned}$

The solution of equation (9) can be expressed in the complex form

$y_{0}=A e^{i \omega_{0} T_{0}}+\bar{A} e^{-i \omega_{0} T_{0}}$ 
where $\bar{A}$ is the complex conjugate of $A$. Then equation (10) becomes,

$$
\begin{aligned}
D_{0}^{2} y_{1}+\omega_{0}^{2} y_{1}= & -\left(3 \lambda A^{2} \bar{A}+i \omega_{0}\left(\mu_{0} A+\mu_{1} A+6 \mu_{1} A^{2} \bar{A}+2 A^{\prime}\right)\right) e^{i \omega_{0} T_{0}} \\
& +F\left(\frac{1}{2}+3 A \bar{A}+15 A^{2} \bar{A}^{2}+70 A^{3} \bar{A}^{3}\right) e^{i \Omega T_{0}} \\
& +F \bar{A}\left(1+6 A \bar{A}+30 A^{2} \bar{A}^{2}\right) e^{i\left(\Omega-\omega_{0}\right) T_{0}} \\
& +F \bar{A}^{2}\left(\frac{3}{2}+10 A \bar{A}+\frac{105}{2} A^{2} \bar{A}^{2}\right) e^{i\left(\Omega-2 \omega_{0}\right) T_{0}} \\
& +F \bar{A}^{3}(2+15 A \bar{A}) e^{i\left(\Omega-3 \omega_{0}\right) T_{0}} \\
& +F \bar{A}^{4}\left(\frac{5}{2}+21 A \bar{A}\right) e^{i\left(\Omega-4 \omega_{0}\right) T_{0}} \\
& +3 F \bar{A}^{5} e^{i\left(\Omega-5 \omega_{0}\right) T_{0}} \\
& +\frac{7}{2} F \bar{A}^{6} e^{i\left(\Omega-6 \omega_{0}\right) T_{0}} \\
& +N S T .+c . c .
\end{aligned}
$$

where NST. denotes the terms which do not produce secular terms and c.c. denotes the complex conjugate.

Equation (12) contain seven cases of resonance

$$
\Omega \simeq n \omega_{0} \quad ; n=1,2,3,4,5,6,7
$$

In this paper we restricted our attention to three cases of resonance (harmonic resonance and subharmonic resonances of orders $\left(\frac{1}{2}\right.$ and $\left.\left.\frac{1}{3}\right)\right)$.

\section{Harmonic resonance $\left(\Omega \simeq \omega_{0}\right)$}

In this section, we study harmonic resonance of the MEMS represented by equation (6). To describe the nearness of the excitation frequency $\Omega$ to the fundamental natural frequency $\omega_{0}$ i.e. $\Omega \simeq \omega_{0}$, we introduce the detuning parameter $\sigma$ to convert the small-divisor terms into secular terms according to

$\Omega=\omega_{0}+\varepsilon \sigma$

Eliminating the secular terms from the equation (12) yields

$-3 \lambda A^{2} \bar{A}-i \omega_{0}\left(\mu_{0} A+\mu_{1} A+6 \mu_{1} A^{2} \bar{A}+2 A^{\prime}\right)+F\left(\frac{1}{2}+3 A \bar{A}+15 A^{2} \bar{A}^{2}+70 A^{3} \bar{A}^{3}\right) e^{i \sigma T_{1}}=0$

Writing $A$ in the polar form as $A=\frac{1}{2} a\left(T_{1}\right) e^{i \beta\left(T_{1}\right)}$ into equation (14) where $a\left(T_{1}\right)$ and $\beta\left(T_{1}\right)$ are real-valued functions, representing, respectively, the amplitude and phase of the response, and separating real and imaginary parts, we obtain the following modulation equations:

$a^{\prime}=-\frac{1}{4} a\left(2 \mu_{0}+\left(2+3 a^{2}\right) \mu_{1}\right)+\frac{\left(16+24 a^{2}+30 a^{4}+35 a^{6}\right) F \sin (\gamma)}{32 \omega_{0}}$

$a \gamma^{\prime}=\sigma a-\frac{3 \lambda a^{3}}{8 \omega_{0}}+\frac{\left(16+24 a^{2}+30 a^{4}+35 a^{6}\right) F \cos (\gamma)}{32 \omega_{0}}$

where $\gamma=\sigma T_{1}-\beta\left(T_{1}\right)$.

For steady state solution, $a^{\prime}=\gamma^{\prime}=0$, in equations (15) and (16) we obtain

$8 \omega_{0} a\left(2 \mu_{0}+\left(2+3 a^{2}\right) \mu_{1}\right)=\left(16+24 a^{2}+30 a^{4}+35 a^{6}\right) F \sin (\gamma)$

$12 \lambda a^{3}-32 \omega_{0} \sigma a=\left(16+24 a^{2}+30 a^{4}+35 a^{6}\right) F \cos (\gamma)$ 
Equations (17) and (18) show that there are no trivial solution at $a=0$. For non-trivial solution i.e. at $a \neq 0$, eliminating $\gamma$ from equations (17) and (18), we get the following frequency- response equation

$64 \omega_{0}^{2} a^{2}\left(2 \mu_{0}+\left(2+3 a^{2}\right) \mu_{1}\right)^{2}+\left(12 \lambda a^{3}-32 \omega_{0} \sigma a\right)^{2}=\left(16+24 a^{2}+30 a^{4}+35 a^{6}\right)^{2} F^{2}$

Solving equation (19) for $\sigma$, we obtain

$\sigma=\frac{12 \lambda a^{3} \pm \sqrt{\left(16+24 a^{2}+30 a^{4}+35 a^{6}\right)^{2} F^{2}-64\left(2 \mu_{0}+\left(2+3 a^{2}\right) \mu_{1}\right)^{2} \omega_{0}^{2} a^{2}}}{32 \omega_{0} a}$

A first-order approximation for the solution of equation (6) can be derived as

$y=a \quad \cos (\Omega t-\gamma)+O(\epsilon)$.

To determined the stability of the non-trivial solutions, let

$a=a_{0}+a_{1}\left(T_{1}\right) \quad \& \quad \gamma=\gamma_{0}+\gamma_{1}\left(T_{1}\right)$

where $a_{0}$ and $\gamma_{0}$ are solutions of equations (17) and (18), and $a_{1}$ and $\gamma_{1}$ are perturbations which are assumed to be small compared with $a_{0}$ and $\gamma_{0}$. Substituting (22) into equations (15) and (16) and linearizing the resulting equations, we obtain that

$a_{1}^{\prime}=\frac{\left(2 \mu_{0} g_{3}+\mu_{1} g_{4}\right)}{4 g_{1}} a_{1}+\left(-\sigma a_{0}+\frac{3 \lambda a_{0}^{3}}{8 \omega_{0}}\right) \gamma_{1}$

$\gamma_{1}^{\prime}=\frac{\left(9 \lambda g_{2} a_{0}^{2}-8 \sigma \omega_{0} g_{3}\right)}{8 \omega_{0} g_{1} a_{0}} a_{1}-\frac{1}{4}\left(2 \mu_{0}+\left(2+3 a_{0}^{2}\right) \mu_{1}\right) \gamma_{1}$

where,

$g_{1}=16+24 a_{0}^{2}+30 a_{0}^{4}+35 a_{0}^{6}, \quad g_{2}=-16-8 a_{0}^{2}+10 a_{0}^{4}+35 a_{0}^{6}$,

$g_{3}=-16+24 a_{0}^{2}+90 a_{0}^{4}+175 a_{0}^{6} \quad$ and $\quad g_{4}=-32-96 a_{0}^{2}+108 a_{0}^{4}+440 a_{0}^{6}+315 a_{0}^{8}$.

Seeking a solution for equations $(23)$ and $(24)$ in the form $\left(a_{1}, \gamma_{1}\right)=\left(\Gamma_{1}, \Gamma_{2}\right) e^{\theta T_{1}}$ where $\Gamma_{1}, \Gamma_{2}$ and $\theta$ are constants, one gets

$2 \omega_{0}\left(4 \theta g_{1}-2 \mu_{0} g_{3}-\mu_{1} g_{4}\right) \Gamma_{1}+a_{0} g_{1}\left(-3 \lambda a_{0}^{2}+8 \sigma \omega_{0}\right) \Gamma_{2}=0$

$\left(-9 \lambda a_{0}^{2} g_{2}+8 \sigma \omega_{0} g_{3}\right) \Gamma_{1}+2 \omega_{0} a_{0} g_{1}\left(4 \theta+2 \mu_{0}+\left(2+3 a_{0}^{2}\right) \mu_{1}\right) \Gamma_{2}=0$

For a nontrivial solution, the determinant of the coefficient matrix must vanish. We obtain this condition as

$\theta=\frac{4 \mu_{0} g_{5}+\mu_{1} g_{8}}{4 g_{1}} \pm \frac{\sqrt{27 \lambda^{2} a_{0}^{4} g_{1} g_{2}-16 \omega_{0}\left(12 \lambda \sigma a_{0}^{2} g_{1} g_{5}-\omega_{0}\left(4 \sigma^{2} g_{1} g_{3}+9 a_{0}^{4}\left(g_{6} \mu_{0}+5 \mu_{1} a_{0}^{2} g_{7}\right)^{2}\right)\right)}}{8 \omega_{0} g_{1}}$

where,

$g_{5}=-8+15 a_{0}^{4}+35 a_{0}^{6}, \quad g_{6}=8+20 a_{0}^{2}+35 a_{0}^{4}$,

$g_{7}=4+10 a_{0}^{2}+7 a_{0}^{4} \quad$ and $\quad g_{8}=-32-96 a_{0}^{2}-12 a_{0}^{4}+140 a_{0}^{6}+105 a_{0}^{8}$.

Consequently, a solution is stable if and only if the real parts of both eigenvalues (27) are less than zero.

\section{Subharmonic resonance of order $1 / 2\left(\Omega \simeq 2 \omega_{0}\right)$}

For this type of oscillations resonance, one must have $\Omega \simeq 2 \omega_{0}$, to express this nearness, let

$\Omega=2 \omega_{0}+\epsilon \sigma$ 
and write

$\left(\Omega-\omega_{0}\right) T_{0}=\omega_{0} T_{0}+\epsilon \sigma T_{0}=\omega_{0} T_{0}+\sigma T_{1}$

Eliminating the secular terms from the equation (12) yields

$-3 \lambda A^{2} \bar{A}-i \omega_{0}\left(\mu_{0} A+\mu_{1} A+6 \mu_{1} A^{2} \bar{A}+2 A^{\prime}\right)+F \bar{A}\left(1+6 A \bar{A}+30 A^{2} \bar{A}^{2}\right) e^{i \sigma T_{1}}=0$

Using the polar form $A=\frac{1}{2} a\left(T_{1}\right) e^{i \beta\left(T_{1}\right)}$ into the equation (30) and separating real and imaginary parts, we obtain the following modulation equations:

$a^{\prime}=-\frac{\left(\mu_{0}+\mu_{1}\right) a}{2}-\frac{3 \mu_{1} a^{3}}{4}+\left(\frac{F a}{2 \omega_{0}}+\frac{3 F a^{3}}{4 \omega_{0}}+\frac{15 F a^{5}}{16 \omega_{0}}\right) \sin (\gamma)$

$a \gamma^{\prime}=\sigma a-\frac{3 \lambda a^{3}}{4 \omega_{0}}+\left(\frac{F a}{\omega_{0}}+\frac{3 F a^{3}}{2 \omega_{0}}+\frac{15 F a^{5}}{8 \omega_{0}}\right) \cos (\gamma)$

where $\gamma=\sigma \mathrm{T}_{1}-2 \beta$.

For steady state solution, $a^{\prime}=\gamma^{\prime}=0$, in equations (31) and (32) we obtain

$$
\begin{aligned}
& \left(\frac{F a}{\omega_{0}}+\frac{3 F a^{3}}{2 \omega_{0}}+\frac{15 F a^{5}}{8 \omega_{0}}\right) \sin (\gamma)=\left(\mu_{0}+\mu_{1}\right) a+\frac{3 \mu_{1} a^{3}}{2} \\
& \left(\frac{F a}{\omega_{0}}+\frac{3 F a^{3}}{2 \omega_{0}}+\frac{15 F a^{5}}{8 \omega_{0}}\right) \cos (\gamma)=-\sigma a+\frac{3 \lambda a^{3}}{4 \omega_{0}}
\end{aligned}
$$

Equations (33) and (34) show that there are two possibilities : (trivial solution ) at $a=0$ and ( nontrivial solution) at $a \neq 0$. Squaring and adding (33) and (34) we get the frequency-response equation

$\sigma=\frac{6 \lambda \omega_{0} a^{2} \pm \sqrt{\left(8+12 a^{2}+15 a^{4}\right)^{2} F^{2} \omega_{0}^{2}-16\left(2 \mu_{0}+\left(2+3 a^{2}\right) \mu_{1}\right)^{2} \omega_{0}^{4}}}{8 \omega_{0}^{2}}$

A first-order approximation for the solution of equation (6) can be derived as

$y=a \quad \cos \left(\frac{1}{2} \Omega t-\frac{1}{2} \gamma\right)+O(\epsilon)$

The analysis of the stability of the trivial solutions is equivalent to the analysis of the linear solutions of equation (30) by neglecting the non-linear terms we get

$-i \omega_{0}\left(\mu_{0} A+\mu_{1} A+2 A^{\prime}\right)+e^{i \sigma T_{1}} F \bar{A}=0$

To solve equation (37) one lets $A=e^{\frac{1}{2} i \sigma T_{1}}\left(B\left(T_{1}\right)+i b\left(T_{1}\right)\right)$ where $\mathrm{B}$ and b are real, separates real and imaginary parts and get

$B\left(F+\sigma \omega_{0}\right)+b \omega_{0}\left(\mu_{0}+\mu_{1}\right)+2 \omega_{0} b^{\prime}=0$

$b\left(-F+\sigma \omega_{0}\right)+B \omega_{0}\left(-\mu_{0}-\mu_{1}\right)-2 \omega_{0} B^{\prime}=0$

Equations (38) and (39) admit solution of the form $(B, b) \propto(B, b) e^{\theta_{0} T_{1}}$ where $B$ and $b$ are constants then

$B\left(F+\sigma \omega_{0}\right)+b \omega_{0}\left(2 \theta_{0}+\mu_{0}+\mu_{1}\right)=0$

$b\left(-F+\sigma \omega_{0}\right)-B \omega_{0}\left(2 \theta_{0}+\mu_{0}+\mu_{1}\right)=0$

the eigenvalues equation can be obtained as

$\theta_{0}=\frac{-\left(\mu_{0}+\mu_{1}\right)}{2} \pm \frac{\sqrt{F^{2}-\sigma^{2} \omega_{0}^{2}}}{2 \omega_{0}}$ 
To analyze the nontrivial solution we followed steps similar to those in the preceding section, we obtain the following variational equations describing the stability of the steady state solutions:

$a_{1}^{\prime}=\frac{3 a_{0}^{2}\left(4 \mu_{0}\left(2+5 a_{0}^{2}\right)+5 \mu_{1} a_{0}^{2}\left(4+3 a_{0}^{2}\right)\right)}{2\left(8+12 a_{0}^{2}+15 a_{0}^{4}\right)} a_{1}+\frac{1}{8} a_{0}\left(-4 \sigma+\frac{3 \lambda a_{0}^{2}}{\omega_{0}}\right) \gamma_{1}$

$\gamma_{1}^{\prime}=\frac{3 a_{0}\left(\lambda\left(-8+15 a_{0}^{4}\right)-8 \sigma \omega_{0}\left(2+5 a_{0}^{2}\right)\right)}{2\left(8+12 a_{0}^{2}+15 a_{0}^{4}\right) \omega_{0}} a_{1}-\left(\mu_{0}+\mu_{1}+\frac{3}{2} a_{0}^{2} \mu_{1}\right) \gamma_{1}$

Equations (43) and (44) admit solution of the form $\left(a_{1}, \gamma_{1}\right)=\left(c_{1}, c_{2}\right) e^{\theta T_{1}}$ where $c_{1}$ and $c_{2}$ are constants, provided that

$\theta=\frac{1}{4}\left(\frac{2\left(d_{2} \mu_{0}-d_{3} \mu_{1}\right)}{d_{1}} \pm \sqrt{\frac{9 a^{4} \lambda^{2} d_{4}-12 a^{2} \lambda \sigma d_{5} \omega_{0}+4\left(24 a^{2} \sigma^{2} d_{6}+d_{7}^{2} \mu_{0}^{2}+2 d_{8} \mu_{0} \mu_{1}+d_{9}^{2} \mu_{1}^{2}\right) \omega_{0}^{2}}{d_{1}^{2} \omega_{0}^{2}}}\right)$

where

$d_{1}=8+12 a^{2}+15 a^{4}, \quad d_{2}=-8+15 a^{4}, \quad d_{3}=8+24 a^{2}+3 a^{4}, \quad d_{4}=-64-96 a^{2}+180 a^{6}+225 a^{8}$, $d_{5}=-64+384 a^{4}+720 a^{6}+675 a^{8}, \quad d_{6}=16+64 a^{2}+90 a^{4}+75 a^{6}, \quad d_{7}=8+24 a^{2}+45 a^{4}$, $d_{8}=64+384 a^{2}+1440 a^{4}+2952 a^{6}+3915 a^{8}+2025 a^{10} \quad$ and $\quad d_{9}=8+24 a^{2}+63 a^{4}+45 a^{6}$.

Consequently, a solution is stable if and only if the real parts of both eigenvalues (45) are less than zero.

\section{Subharmonic resonance of order $1 / 3\left(\Omega \simeq 3 \omega_{0}\right)$}

In this case, $\Omega \simeq 3 \omega_{0}$. Then we can write

$\Omega=3 \omega_{0}+\epsilon \sigma$

and write

$\left(\Omega-2 \omega_{0}\right) T_{0}=\omega_{0} T_{0}+\epsilon \sigma T_{0}=\omega_{0} T_{0}+\sigma T_{1}$

Then eliminating the secular terms from the equation (12) yields

$-3 \lambda A^{2} \bar{A}-i \omega_{0}\left(\mu_{0} A+\mu_{1} A+6 \mu_{1} A^{2} \bar{A}+2 A^{\prime}\right)+F \bar{A}^{2}\left(\frac{3}{2}+10 A \bar{A}+\frac{105}{2} A^{2} \bar{A}^{2}\right) e^{i \sigma T_{1}}=0$

Using the polar form $A=\frac{1}{2} a\left(T_{1}\right) e^{i \beta\left(T_{1}\right)}$ into the equation (48) and separating real and imaginary parts, we obtain the following modulation equations:

$a^{\prime}=-\frac{\left(\mu_{0}+\mu_{1}\right) a}{2}-\frac{3 \mu_{1} a^{3}}{4}+\left(\frac{3 F a^{2}}{8 \omega_{0}}+\frac{5 F a^{4}}{8 \omega_{0}}+\frac{105 F a^{6}}{128 \omega_{0}}\right) \sin (\gamma)$

$a \gamma^{\prime}=\sigma a-\frac{9 \lambda a^{3}}{8 \omega_{0}}+3\left(\frac{3 F a^{2}}{8 \omega_{0}}+\frac{5 F a^{4}}{8 \omega_{0}}+\frac{105 F a^{6}}{128 \omega_{0}}\right) \cos (\gamma)$

where $\gamma=\sigma \mathrm{T}_{1}-3 \beta$.

For steady state solution, $a^{\prime}=\gamma^{\prime}=0$, in equations (49) and (50) we obtain

$$
\begin{aligned}
& \left(3 F a^{2}+5 F a^{4}+\frac{105 F a^{6}}{16}\right) \sin (\gamma)=6 \mu_{1} \omega_{0} a^{3}+4 \omega_{0} a\left(\mu_{0}+\mu_{1}\right) \\
& \left(3 F a^{2}+5 F a^{4}+\frac{105 F a^{6}}{16}\right) \cos (\gamma)=3 \lambda a^{3}-\frac{8}{3} \sigma \omega_{0} a
\end{aligned}
$$

Equations (51) and (52) show that there are two possibilities : (trivial solution ) at $a=0$ and ( nontrivial solution) at $a \neq 0$. Squaring and adding (51) and (52) we get the frequency-response equation

$\sigma=\frac{3\left(48 \lambda \omega_{0} a^{2} \pm \sqrt{F^{2} \omega_{0}^{2}\left(48 a+80 a^{3}+105 a^{5}\right)^{2}-1024 \omega_{0}^{4}\left(\left(3 a^{2}+2\right) \mu_{1}+2 \mu_{0}\right)^{2}}\right)}{128 \omega_{0}^{2}}$ 
A first-order approximation for the solution of equation (6) can be derived as

$y=a \quad \cos \left(\frac{1}{3} \Omega t-\frac{1}{3} \gamma\right)+O(\epsilon)$

Now, the analysis of the stability of the trivial solution is determined as in the preceding section, so we get the eigenvalues equation obtained as

$\theta_{0}=-\frac{1}{2}\left(\mu_{0}+\mu_{1}\right) \pm \frac{1}{2} i \sigma$

Also, the stability of the nontrivial solution is determined similar to the preceding section and the eigenvalues equation can be obtained as

$\theta=\frac{12\left(\mu_{0} z_{2}-\mu_{1} z_{3}\right)}{8 z_{1}} \pm \sqrt{\frac{27 \lambda^{2} a^{4} z_{4}-960 \lambda \sigma \omega_{0} a^{4} z_{5}+4\left(16 \sigma^{2} z_{6}+576 \mu_{0}^{2} z_{7}^{2}+144 \mu_{0} \mu_{1} z_{8}+9 \mu_{1}^{2} z_{9}^{2}\right) \omega_{0}^{2}}{64 \omega_{0}^{2} z_{1}^{2}}}$

where

$z_{1}=48+80 a^{2}+105 a^{4}, \quad z_{2}=-16+35 a^{4}, \quad z_{3}=16+48 a^{2}+5 a^{4}, \quad z_{4}=-2304+16480 a^{4}+33600 a^{6}+33075 a^{8}$, $z_{5}=384+1648 a^{2}+2520 a^{4}+2205 a^{6}, \quad z_{6}=768+5120 a^{2}+16480 a^{4}+22400 a^{6}+18375 a^{8}, \quad z_{7}=8+20 a^{2}+35 a^{4}$, $z_{8}=512+2944 a^{2}+9920 a^{4}+18600 a^{6}+21700 a^{8}+11025 a^{10} \quad$ and $\quad z_{9}=64+208 a^{2}+440 a^{4}+315 a^{6}$.

Consequently, a solution is stable if and only if the real parts of both eigenvalues (56) are less than zero.

\section{Numerical results and discussion}

This section presents numerical results in the form of frequency response curves obtained by solving the frequency response equations (20), (35), (53) and stability conditions (27), (42), (45), (55) and (56). The numerical results are plotted in groups of figures (2-7), (8-14) and (15-21), which represent the variation of the amplitude $(a)$ with the detuning parameter $(\sigma)$ for given values of the other parameters. In all figures, the solid lines represent stable solutions, while the dashed lines represent unstable solutions.

Figures (2-7) represent the frequency response curves for the harmonic resonance for certain values of the parameters. In (Fig.2) we have two semi-symmetric ovals about $\sigma=0$. The lower values of $a$ are stable solutions and the upper values of $a$ are unstable solutions.

- By increasing (decreasing) the coefficient of the parametric and external force $F$ (amplitude of the excitation) the region of definition is decreased (increased), we have also by decreasing $F$ two continuous solutions lower branch is stable and the upper branch is unstable (fig.3).

- By decreasing (increasing) the value of natural frequency $\omega_{0}$ the region of definition is decreased (increased), also by increasing $\omega_{0}$ we have two continuous solutions lower branch is stable and the upper branch is unstable (fig.4).

- By increasing the value of $\lambda$, the symmetry is disappear and the value of unstable solution is increased (fig.5).

- By decreasing (increasing) the value of $\mu_{0}$ or $\mu_{1}$ the region of definition is decreased (increased), also by increasing $\mu_{0}$ or $\mu_{1}$ we have two continuous solutions lower branch is stable and the upper branch is unstable (fig.6, fig.7).

- By increasing the value of $\sigma$ after certain value we note that their is no changes in the magnitudes for stable and unstable solutions for given values of $\mu_{0}$ (i.e. there exists a saturation) (fig.6), while their exist a saturation in the stable solutions only for given values of $\mu_{1}$ (fig.7).

Figures (8-14) represent the frequency response curves of the subharmonic resonance of order $\frac{1}{2}$. In all figures (8-14) the trivial solution is stable. When we consider the same values of the parameters in the harmonic resonance, we get a continues unstable curve (Fig.8), so we consider other values of the parameters. In (Fig.9) we have two curves, the right curve has unstable solutions while the left curve has a multi-value solutions with the upper branch is unstable, the lower branch is stable and there exist a saddle node bifurcation. 
- By increasing $F$, the region of definition and the magnitudes of the solutions are decreased. When $F=1.5$ the stable solution disappears. By decreasing $F$, we have a one continues curve with jumping phenomenon (fig.10).

- By increasing (decreasing) $\omega_{0}$, the region of definition and the magnitudes of the solutions are increased (decreased). For $\omega_{0}=0.9$ we have an unstable curve (fig.11).

- By increasing (decreasing) $\lambda$, the region of definition, the magnitudes of the solutions and the region of stability of the left curve are increased (decreased), while the magnitudes of the solutions of the right curve is decreased (increased). For $\lambda=1.0$ we have two unstable curves (fig.12).

- By increasing (decreasing) $\mu_{0}$ or $\mu_{1}$, the region of definition and the magnitudes of the solutions are increased (decreased). As $\mu_{0}=2.0$ or $\mu_{1}=1.8$, the response amplitude loses stability and we have an unstable curve (fig.13, fig.14).

Figures (15-21) represent the frequency response curves of the subharmonic resonance of order $\frac{1}{3}$. In all figures (15-21) the trivial solution is stable. When we consider the same values of the parameters in the harmonic resonance, we get a continues unstable curve (Fig.15), so we consider other values of the parameters. In (Fig.16) we have a continues curve with region of multivalued solutions, where the middle solution is stable and the upper and the lower solutions are unstable.

- By decreasing (increasing) $F$, we note that the magnitude of (a), the region of multivalued and the region of stability are increased (decreased). As $F=1.4$, the response amplitude loses stability and we have a one continues unstable curve (fig.17).

- By decreasing (increasing) $\omega_{0}$, the region of multivalued and stability is increased (decreased) and the magnitude of (a)is decreased (increased). When $\omega_{0}=1.4$, the response amplitude loses stability and the region of multivalued is disappeared. Also, we can see that there exist intersection point at $\sigma=0.0$ (fig.18).

- By decreasing (increasing) $\lambda$, the magnitude of (a) for the upper solutions and the region of multivalued and stability are decreased (increased), but, the magnitude of (a) for the lower solutions is increased (decreased). When $\lambda=1.0$, the response amplitude loses stability and the region of multivalued is disappeared (fig.19).

- By decreasing (increasing) $\mu_{0}$ or $\mu_{1}$, the region of multivalued and stability is increased (decreased). For $\mu_{0}=1.0$ or $\mu_{1}=0.6$, the response amplitude loses stability and we have a continues unstable curve (fig.20, fig.21). 


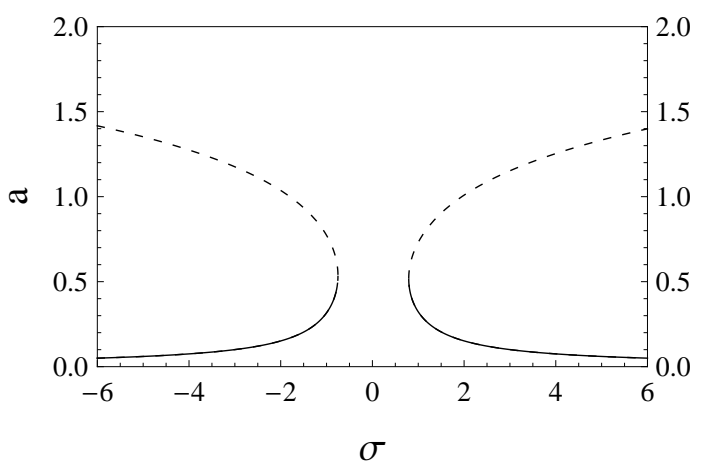

Fig.2: The frequency response curves of the harmonic resonance for the parameters $F=0.3, \omega_{0}=0.5, \lambda=0.1, \mu_{0}=0.7$ and $\mu_{1}=0.2$

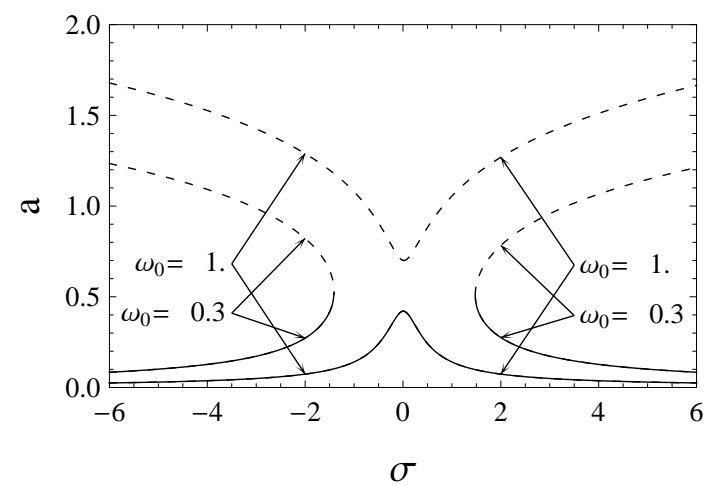

Fig.4: Variation of the amplitude of the response with the detuning parameter for increasing and decreasing $\omega_{0}$

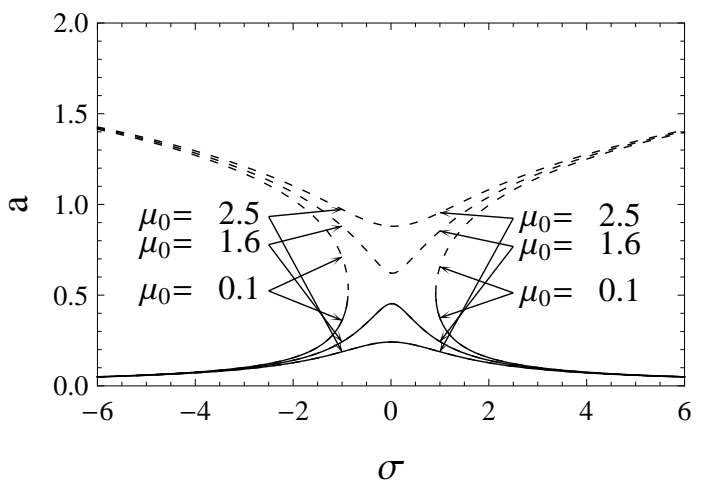

Fig.6: Variation of the amplitude of the response with the detuning parameter for increasing and decreasing $\mu_{0}$

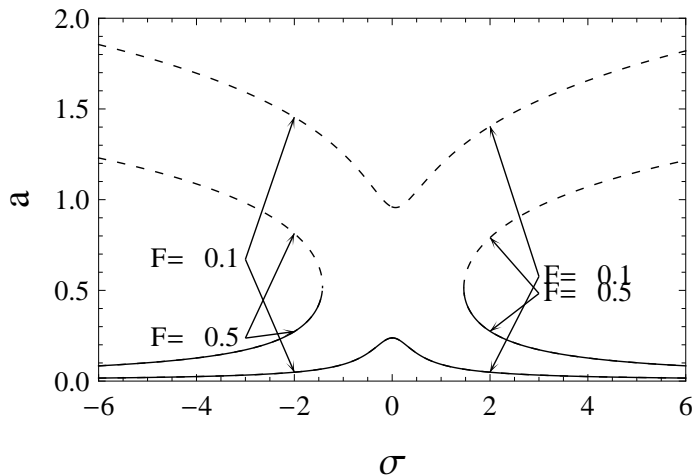

Fig.3: Variation of the amplitude of the response with the detuning parameter for increasing and decreasing $F$

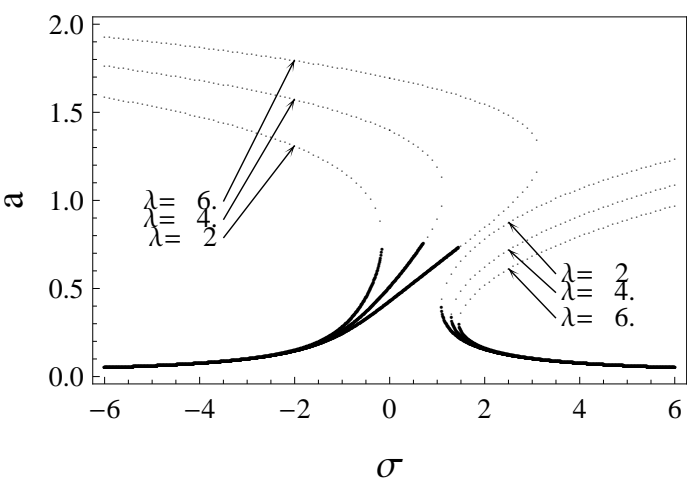

Fig.5: Variation of the amplitude of the response with the detuning parameter for increasing and decreasing $\lambda$

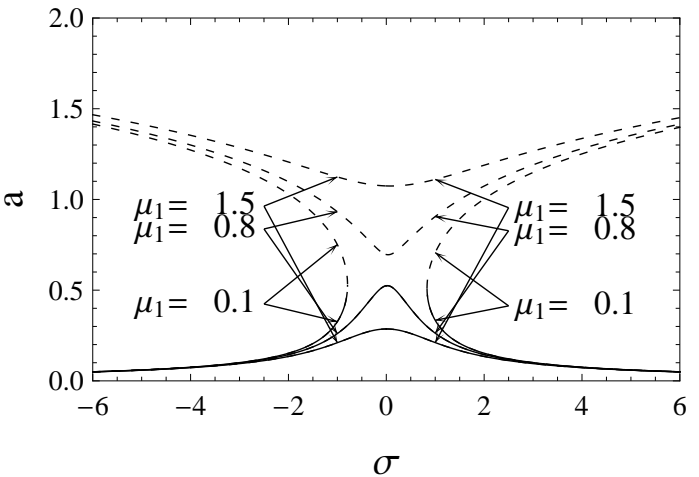

Fig.7: Variation of the amplitude of the response with the detuning parameter for increasing and decreasing $\mu_{1}$ 


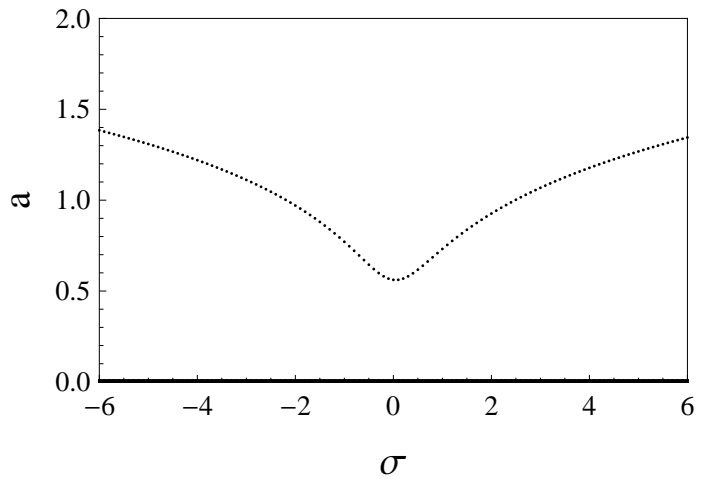

Fig.8: The frequency response curves of the subharmonic resonance of order $\frac{1}{2}$ for the parameters $F=0.3$ $\omega_{0}=0.5, \lambda=1, \mu_{0}=0.7$ and $\mu_{1}=0.2$

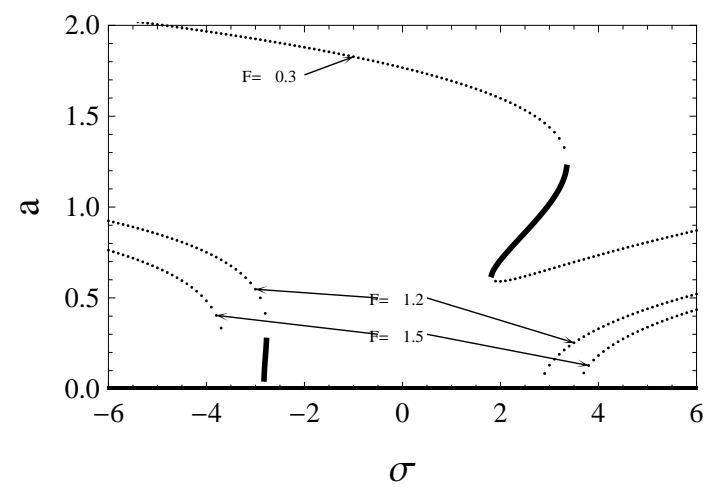

Fig.10: Variation of the amplitude of the response with the detuning parameter for increasing and decreasing $F$

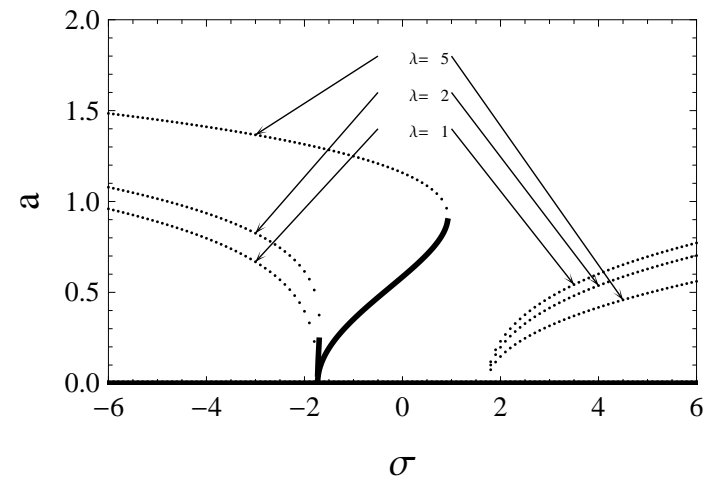

Fig.12: Variation of the amplitude of the response with the detuning parameter for increasing and decreasing $\lambda$

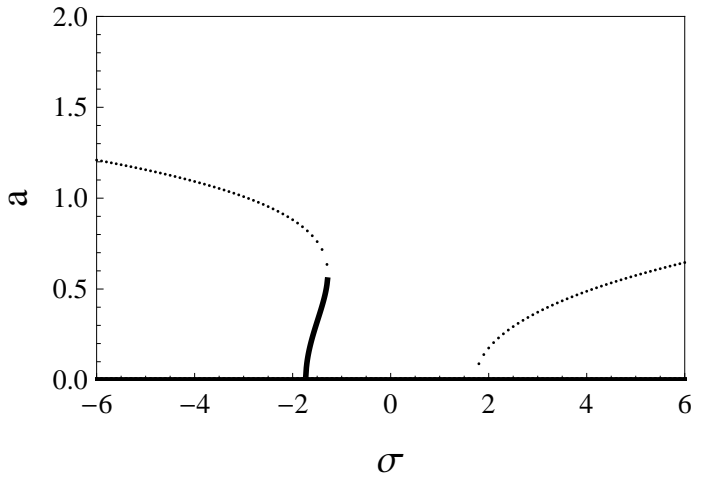

Fig.9: The frequency response curves of the subharmonic resonance of order $\frac{1}{2}$ for the parameters $F=0.8$ $\omega_{0}=0.4, \lambda=3, \mu_{0}=0.4$ and $\mu_{1}=0.6$

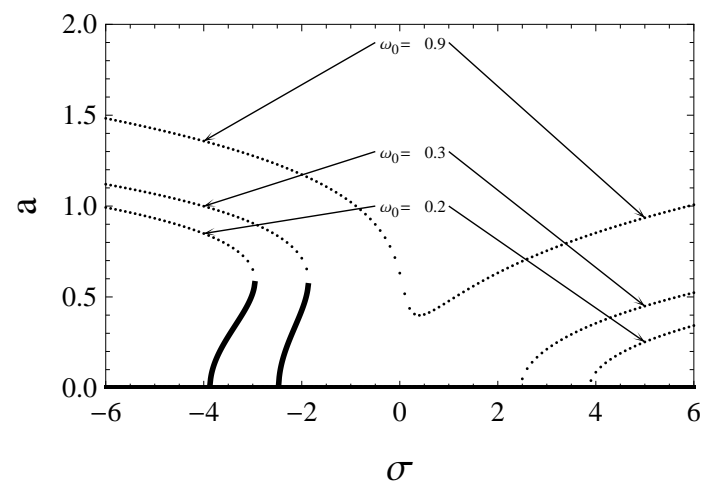

Fig.11: Variation of the amplitude of the response with the detuning parameter for increasing and decreasing $\omega_{0}$

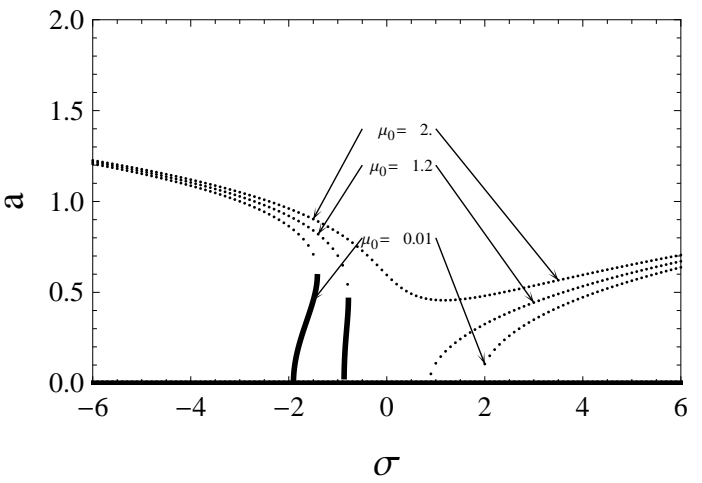

Fig.13: Variation of the amplitude of the response with the detuning parameter for increasing and decreasing $\mu_{0}$ 


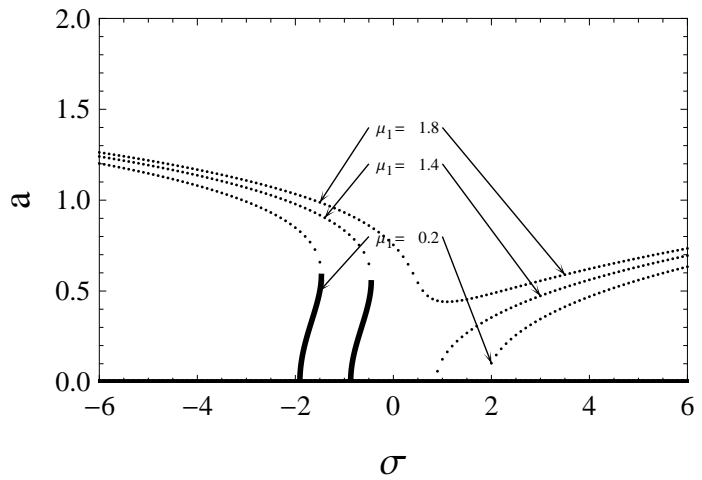

Fig.14: Variation of the amplitude of the response with the detuning parameter for increasing and decreasing $\mu_{1}$

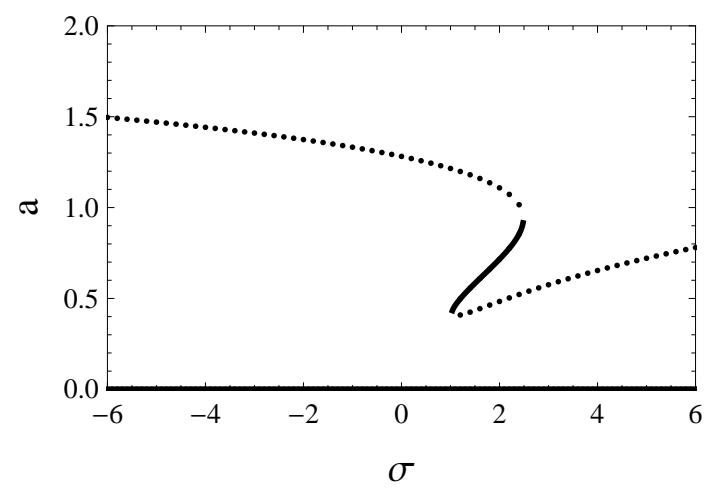

Fig.16: The frequency response curves of the subharmonic resonance of order $\frac{1}{3}$ for the parameters $F=0.4$ $\omega_{0}=0.5, \lambda=3.0, \mu_{0}=0.2$ and $\mu_{1}=0.1$

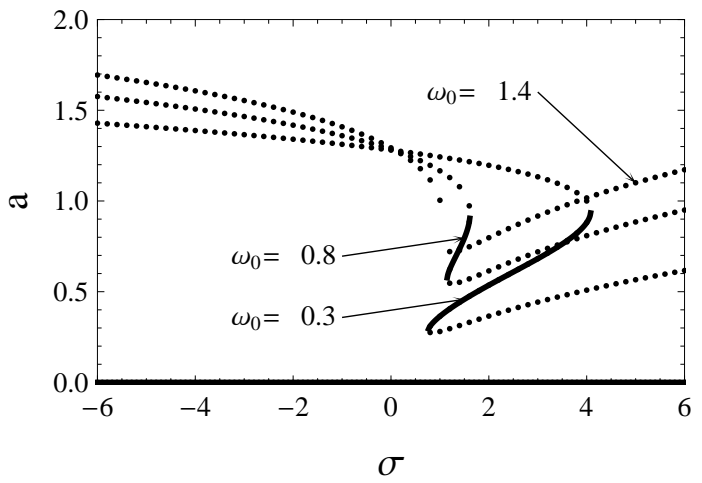

Fig.18: Variation of the amplitude of the response with the detuning parameter for increasing and decreasing $\omega_{0}$

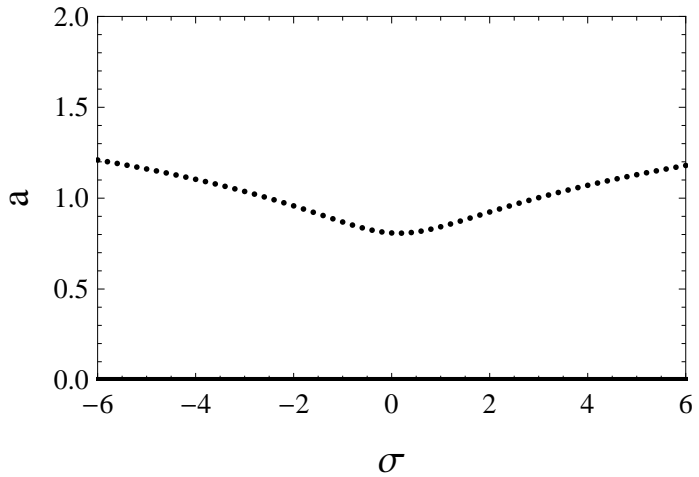

Fig.15: The frequency response curves of the subharmonic resonance of order $\frac{1}{3}$ for $F=0.3, \omega_{0}=0.5, \lambda=1.0, \mu_{0}=0.7, \mu_{1}=0.2$

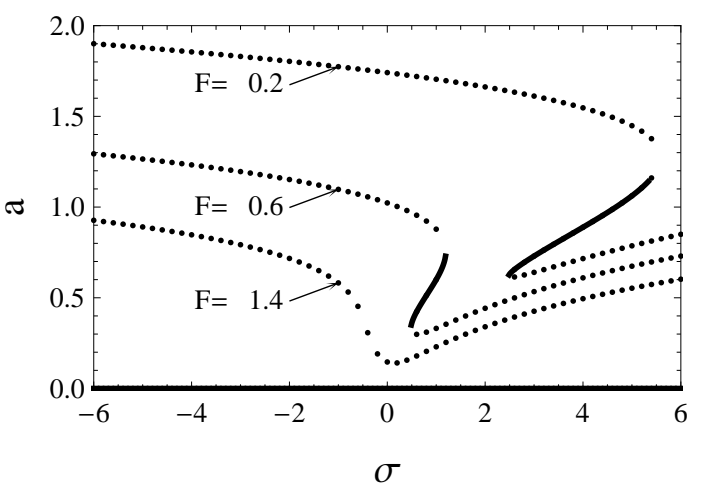

Fig.17: Variation of the amplitude of the response with the detuning parameter for increasing and decreasing $F$

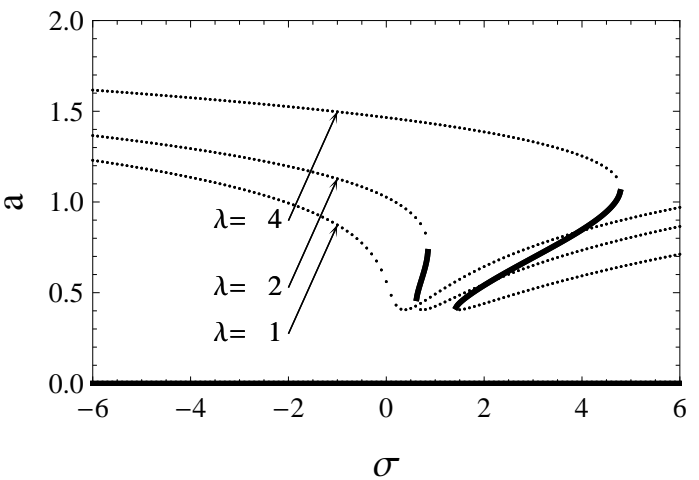

Fig.19: Variation of the amplitude of the response with the detuning parameter for increasing and decreasing $\lambda$ 


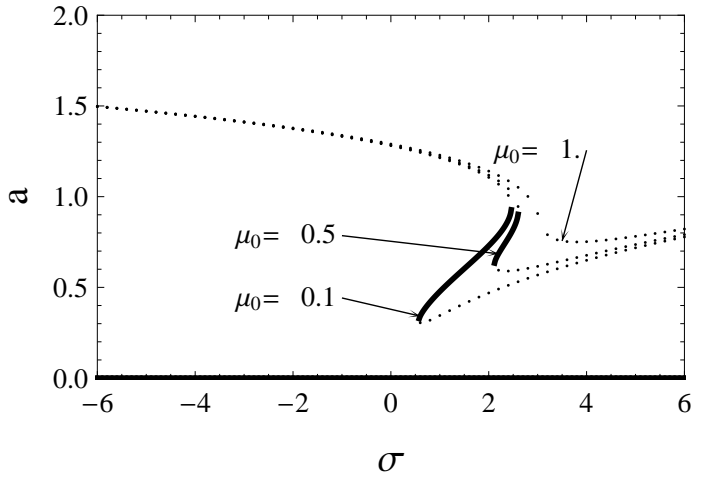

Fig.20: Variation of the amplitude of the response with the detuning parameter for increasing and decreasing $\mu_{0}$

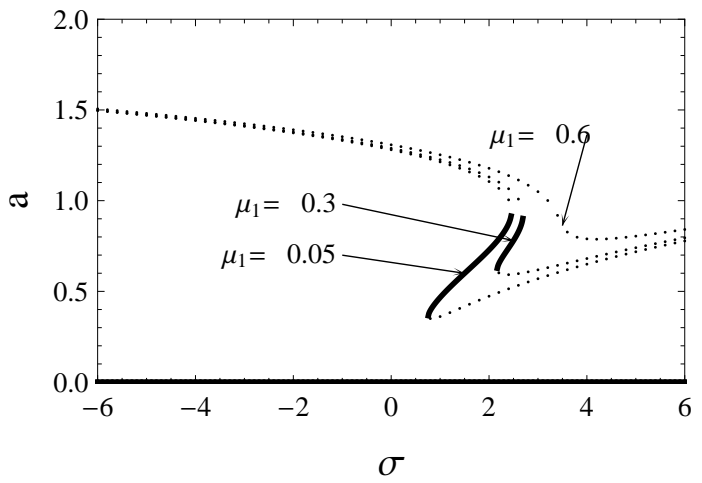

Fig.21: Variation of the amplitude of the response with the detuning parameter for increasing and decreasing $\mu_{1}$

\section{Conclusion}

In this paper we studied the weakly non-linear dynamic response (micro - electro - mechanical systems (MEMS)) represented by a mathematical model with modified Duffing - Van der Pol equation subjected to weakly non-linear parametric and forcing excitations. The study is devoted to harmonic resonance and subharmonic resonances of orders $\left(\frac{1}{2}\right.$ and $\left.\frac{1}{3}\right)$. By using multiple scales method, for each resonance modulation equation, steady-state solutions, frequency response equation and stability of the steady-state solution are determined. Frequency response curves are plotted. Finally discussion for the figures is provided.

From the frequency response curves of the harmonic resonance, we observe that the response amplitude consists of two semi-symmetric ovals about $\sigma=0$, the lower values of the amplitude $(a)$ are stable solutions and the upper values of the amplitude $(a)$ are unstable solutions. By increasing $F$ or decreasing any of $\omega_{0}, \mu_{0}$ or $\mu_{1}$, we get the region of definition is decreased. While, by decreasing $F$ or increasing any of $\omega_{0}, \mu_{0}$ or $\mu_{1}$, we get two continuous curves, the lower curve has stable solutions and the upper curve has unstable solutions. By increasing the value of $\lambda$, the symmetry disappears and the region of stability is increased.

From the frequency response curves of the subharmonic resonance of order $\frac{1}{2}$ we observe that the trivial solution is stable. The non trivial solutions loses stability and the region of multivalued disappear by increasing any of $\omega_{0}$, $\mu_{0}$ or $\mu_{1}$. The regions of definition are increased (decreased) when the values of the parameters $\omega_{0}, \mu_{0}$ or $\mu_{1}$ are increased (decreased). When increasing $F$ or decreasing $\lambda$ we get two unstable curves. By increasing the value of $\lambda$ the region of multivalued and stability is increased.

From the frequency response curves of the subharmonic resonance of order $\frac{1}{3}$ we observe that the trivial solution has stable solutions. The non trivial solutions loses stability and the region of multivalued disappear by decreasing $\lambda$ or increasing any of $F, \omega_{0}, \mu_{0}$ or $\mu_{1}$. The regions of multivalued and stability is increased (decreased) when the values of the parameters $F, \omega_{0}, \mu_{0}$ or $\mu_{1}$ are decreased (increased). By increasing the value of $\lambda$ the region of multivalued and stability is increased.

\section{References}

[1] W.C. Chuang, H.L. Lee, P.Z. Chang and Y.C. Hu, Review on the Modeling of Electrostatic MEMS, Sensors, 10 (6)(2010) 6149-6171.

[2] S. Pamidighantam, R. Puers, K. Baert, and A.C. Tilmans, Pull-in voltage analysis of electrostatically actuated beam structures with fixed-fixed and fixed-free end conditions, J. Micromech. Microeng,12 (2002) 454-464.

[3] T. Sasayama, S. Suzuki, S. Tsuchitani, A. Koide, M. Suzuki, N. Ichikawa and T. Nakazawa, Highly reliable silicon micromachined physical sensors in mass production, Sensors and Actuators A,54 (1996) 714-717.

[4] M.H. Bao, H. Yang, H. Yin and S.Q. Shen, Effects of electrostatic forces generated by the driving signal on capacitive sensing devices, Sensors and Actuators A,24 (2000) 213219.

[5] S. Lee, R. Ramadoss, M. Buck, V.M. Bright, K.C. Gupta and Y.C. Lee, Reliability testing of flexible printed circuitbased RF MEMS capacitive switches, Microelectronics Reliability,44 (2004) 245250. 
[6] B. MoCarthy, G.G. Adams, N.E. McGruer and D. Potter, A dynamical model, including contact bounce of an electrostatically actuated microswitch, J Microelectromech Syst,11 (2002) 276-283.

[7] M. Mehregany, P. Nagarkar, S.D. Senturia and J.H. Lang, Operation of microfabricated harmonic and ordinary side-drive motors, Proc. 3rd. IEEE MEMS Workshop, Napa Valley, CA, (1990) 1-8.

[8] L.S. Tavrow, S.F. Bart and J.H. Lang, Operational characteristics of microfabricated electric motors, Sensors and Actuators A, 35 (1992) 33-44.

[9] E.S. Hung S.D. Senturia, Extending the travel range of analog-tuned electrostatic actuators, J. Microelectromech Syst,8 (4) (1999) 497-505.

[10] P. Osterberg, Electrostatically actuated microelectromechanical test structures for material property measurement, PhD thesis, MIT (1995).

[11] M.I. Younis , E.M. Abdel-Rahman and A.H. Nayfeh, Static and dynamic behavior of an electrically excited resonant microbeam, Proceedings of the 43rd AIAA structures, structural dynamics, and materials conference. Denver, CO, (2002) AIAA Paper 2002/1305.

[12] E.M. Abdel-Rahman, M.I. Younis and A.H. Nayfeh, A nonlinear reduced-order model for electrostatic MEMS, Proceedings of the 19th biennial conference in mechanical vibration and noise (VIB). Chicago, IL, (2003) Paper DETC2003/VIB48517.

[13] J.A. Walraven, Future challenges for MEMS failure analysis, Proceedings of ITC international test conference, 33 (4) (2003) 850-5.

[14] E.M. Abdel-Rahman, M.I. Younis and A.H. Nayfeh, Characterization of the mechanical behavior of an electrically actuated microbeam, J. Micromech Microeng, 12 (2002) 766-95.

[15] H.A. Tilmans and R. Legtenberg, Electrostatically driven vacuum-encapsulated polysilicon resonators, Part II: theory and performance, Sensors Actuators A, 45 (1994) 67-84.

[16] M.I. Younis, E.M. Abdel-Rahman and A.H. Nayfeh, Dynamic simulations of a novel RF MEMS switch, Proceedings rth Int. Conf. Modeling and Simulation of Microsystems: NanoTech, Boston, MA, (2004) 287-290.

[17] L. Lin, C.T.-C. Nguyen, R.T. Howe and A.P. Pisano, Microelectromechanical filters for signal processing, Proceedings IEEE MEMS, Travemunde, Germany, (1992) 226-231.

[18] Z. Jin and Y. Wang, Electrostatic resonator with second superharmonic resonance, Sens. Actuators A, 64 (1998) $273-279$.

[19] M.I. Younis and A.H. Nayfeh, A study of the nonlinear response of a resonant microbeam to electric actuation, Nonlinear Dyn., 31 (2003) 91-117.

[20] E.M. Abdel-Rahman and A.H. Nayfeh, Secondary resonances of electrically actuated resonant microsensors, Journal of Micromechanics and Microengineering, 13 (2003) 491-501.

[21] A.H. Nayfeh and M.I. Younis, Dynamics of MEMS resonators under superharmonic and subharmonic excitations, J. of Micromechanics and Microengineering, 15 (2005) 1840-1847.

[22] W.M. Zhang and G. Meng, Nonlinear Dynamic Analysis of Electrostatically Actuated Resonant MEMS Sensors Under Parametric Excitation. IEEE sensors journal, 7 (2007) 370-380.

[23] W.M. Zhang, G. Meng and K.X. Wei, Dynamics of nonlinear coupled electrostatic micromechanical resonators under two frequency parametric and external excitations, Shock and Vibration, 17 (2010) 759-770.

[24] A.M. Elnaggar, Existence and Determination of Superharmonic Synchronizations as Solution,of a Quasi-Linear Physical System, Indian. J.Pure. Appl. Math., 16 (1985) 139-142.

[25] A.M. Elnaggar, A.F. El-Bassiouny and K.M. Khalil, Saddle-node bifurcation control for an odd non-linearity problem, Global J. of Pure and Applied Mathematics, 7 (2011) 213-229.

[26] A.M. Elnaggar, A.F. El-Bassiouny and G.A. Mosa, Harmonic and sub-harmonic resonance of MEMS subjected to a weakly non-linear parametric and external excitations, International Journal of Applied Mathematical Research (IJAMR), North America, 2 (2) (2013) 252-263.

[27] A.M. Elnaggar and K.M. Khalil, Control of the nonlinear oscillator bifurcation under a superharmonic resonance. Journal of Applied Mechanics and Technical Physics, 54 (1) (2013) 34-43. 
[28] R.C. Batra, M. Porfiri and D. Spinello, Vibrations of narrow micro beams predeformed by an electric field, Journal of Sound and Vibration, 309 (3) (2008) 600-612.

[29] N. Kacem, S. Hentz, D. Pinto, B. Reig and V. Nguyen, Nonlinear dynamics of nanomechanical beam resonators: improving the performance of NEMS- based sensor, Nanotechnology,20 (27) (2009) 275501.

[30] N. Kacem, S. Hentz, H. Fontaine, V. Nguyen, P. Robert, B. Legrand and L. Buchaillot, From MEMS to NEMS: modelling and characterization of the non linear dynamics of resonators, a way to enhance the dynamic range, NSTI Nanotech, 3 (2008) 619-622

[31] N. Kacem, S. Baguet, S. Hentz and R. Dufour, Computational and quasi-analytical models for non-linear vibrations of resonant MEMS and NEMS sensors, International Journal of Non-Linear Mechanics, 46 (3) (2011) 532-542.

[32] J.F. Rhoads, S.W. Shaw and K.L. Turner, Non-linear dynamics and its applications in micro- and nanoresonators, J. Dyn. Syst. Meas. Control, 132 (2010) 034001 (1-034001.14).

[33] K.L. Turner, P.G. Hartwell, F.M. Bertsch and N.C. Macdonald, Parametric resonance in a microelectromechanical torsional oscillator, Proceedings of ASME International Mechanical Engineering Congress and Exposition, Proceedings of the Microelectromechanical Systems (MEMS), Anaheim, CA, USA, (1998) 335-340.

[34] J.B. Starr, Squeeze-film damping in solid-state accelerometers, Proceedings IEEE Solid State Sensor and Actuator Workshop, Hilton Head Island, SC, (1990) 44-47.

[35] A.H. Nayfeh, Introduction to Perturbation Techniques, Wiley-Interscience, New York (1981). 